\title{
LECTURE
}

\section{Clinical experience with transdermal fentanyl for the treatment of cancer pain in Germany}

\author{
Lukas Radbruch and Frank Elsner \\ Department of Palliative Medicine, University Hospital Aachen, Aachen, Germany
}

(Received for publication on May 6, 2003)

\begin{abstract}
The transdermal therapeutic system with fentanyl was released in Germany in 1995. Before and after the release several clinical trials were performed in our pain management unit and in other German pain centers, showing good efficacy after initial dose titration with intravenous patientcontrolled analgesia or switching from pretreatment with oral morphine or other opioids. A sequential trial showed less use of laxatives with transdermal fentanyl compared to pretreatment with oral morphine. Safety and efficacy of the transdermal system in clinical practice were confirmed in a nationwide survey with 1005 patients, nearly all of them with cancer pain. Most patients had been treated with opioids, though $22 \%$ had received no opioids or only as required before initiation of transdermal fentanyl. The mean duration of transdermal treatment was $71 \pm 83$ days. Pain relief with transdermal therapy was swift and efficient. Adverse events with the possibility of a causal relationship to transdermal therapy were documented for $26 \%$ of the patients, most frequently nausea, vomiting, constipation and drowsiness. Severe neurotoxic or respiratory complications were reported only rarely. Problems with transdermal application were reported by $12 \%$ of the patients, with patch detachment and dermatologic symptoms on the site of application being most frequent. Most patients showered regularly with the patches and only three patients reported that patches became loose under the shower or in the bathtub. In a recent prospective trial driving ability was tested in patients with continuous non-cancer pain, who had received stable doses of transdermal fentanyl. Data were available from 90 healthy volunteers matched to 30 patients, of whom 9 were excluded from the analysis because they took additional drugs in violation of the protocol. None of the performance measures for the 21 remaining fentanyl patients was significantly inferior to the controls. In conclusion, experience with the transdermal therapeutic system with fentanyl has been gathered in clinical trials, a large nationwide survey and clinical practice since the release in 1995. The conversion table based on a conversion ratio of 100:1 was safe and efficient in trials and clinical practice. Transdermal fentanyl has become a wellknown and frequently used opioid in the treatment of chronic cancer and non-cancer pain in Germany. (Keio J Med 53 (1): 23-29, March 2004)
\end{abstract}

Key words: Transdermal fentanyl, Germany, Cancer pain, Constipation

The application of anaegesics by the oral route is one of the major recommendations of the World Health Organisation in their cancer pain guidelines first published in 1986 (World Health Organisation 1986). ${ }^{1}$ Regular application with fixed intervals and the analgesic ladder using non-opioids for slight pain, codeinetype opioids for moderate pain and morphine-type opioids for severe pain were the other important rec- ommendations in these guidelines. Following these guidelines, oral morphine, preferably with slow release tablets, has become the most important analgesic drug in many countries. The construction of the fentanyl patch, that allowed the continous transdermal application of a potent opioid, has provided new options, and its benefit for example for patients unable to take oral medications is evident.

Presented at the 1309th Meeting of the Keio Medical Society in Tokyo, February 26, 2003.

Reprint requests to: Dr. Lukas Radbruch, Department of Palliative Medicine, University of Aachen, Pauwelsstr. 30, 52074 Aachen, Germany, telephone: ++49 24180 80726, facsimile: ++49 2418033 80726, e-mail: lradbruch@ukaachen.de 


\section{Clinical Trials with Transdermal Fentanyl in Germany}

The fentanyl patch was released in the USA in 1992. In Germany the patch system with fentanyl was released in 1995, though the release was burdened with some restrictions initially. The initiation of the transdermal opioid therapy was allowed only for inpatients with chronic cancer pain and only by pain specialists. After the initial phase continuation of the therapy was possible by other non-specialized physicians and in the out-patient setting as well. The reason for these restrictions were concerns of the authorities about the safety of the transdermal system. Only in 1998 the restrictions were lifted, allowing the initiation of the transdermal therapy also in the out-patient setting and by non-specialists. Another year later, in 1999, transdermal fentanyl was released for the use in chronic noncancer pain as well.

Even before the release in Germany our unit has been involved in the research on transdermal fentanyl. A pilot study with 20 patients (Zech et al. 1992) ${ }^{2}$ and a subsequent trial with another 50 patients (Grond et al. $1997)^{3}$ used intravenous patient-controlled analgesia (PCA) for the initial dose titration of the transdermal therapy. The pilot study used a conversion ratio of $1: 1$ from the 24-hour dosage of the PCA to transdermal fentanyl. With this ratio most patients required dose increases with the transdermal appllication, and so the following study used a conversion ratio of $1: 1.5$. However, with this higher ratio, mild respiratory depression was seen in 3 of the 50 patients. Other side effects were rare, and patients were treated up to 18 months without complications.

In the meantime another clinical multicenter trial in Germany had converted patients pretreated with oral morphine to transdermal fentanyl and had shown good results with a conversion ratio of 100:1 for the daily dosages (for example $60 \mathrm{mg} /$ day morphine $=$ $0.6 \mathrm{mg} /$ day fentanyl = one patch with $2.5 \mathrm{mg}$ ). A conversion table using this ratio of 100:1 was recommended subsequently and was included in the package insert.

One of the advantages of the transdermal therapeutic system in these trials were lower incidences of constipation compared to other oral opioids used for pretreatment. Significantly less constipation with transdermal fentanyl than with oral morphine was found in an open cross-over study with 202 patients from palliative care centers in the United Kingdom (Ahmedzai and Brooks 1997). ${ }^{4}$ One item of the quality of lifequestionnaire of the European Association for the Treatment of Cancer Pain (EORTC) were used to assess constipation. Mean score after 2 weeks of fentanyl therapy was 20.7 compared to 36.6 after 15 days of morphine treatment.
In a German multicenter study by Donner and colleagues 38 patients were treated with slow release morphine for 6 days and then switched to transdermal fentanyl for 15 days (Donner et al. 1996). ${ }^{5}$ Patients reported constipation on $58.8 \%$ of days with morphine. This was reduced to $35.1 \%$ of the days with transdermal fentanyl. Concomitantly, the medication with laxatives was reduced from $62 \%$ to $38 \%$ of days. However, assessment of constipation was not standardised. Side effects such as constipation were elicited by questioning and control of the pain diary.

In a non-randomized sequential cross-over study additional data were compiled on constipation rates and use of laxatives (Radbruch et al. 2000). ${ }^{6}$ In this multicenter trial patients with adequate pain relief from oral morphine documented bowel movements and use of laxatives for six days. Patients then were switched to transdermal fentanyl using the conversion ratio of $100: 1$.

The number of patients with bowel movements did not change after the opioid switch but the number of patients taking laxatives was reduced significantly from $78-87 \%$ of the patients per treatment day (morphine) to $22-48 \%$ (fentanyl). Lactulose was used mainly and was reduced most drastically but other laxatives were used less frequently, too.

In this trial the equianalgesic ratio was calculated after dose adjustments to archive the same level of pain relief after switching to transdermal fentanyl. Comparing the median dose of $2.4 \mathrm{mg} /$ day on day 15 with the median morphine dosage before conversion, a ratio of $75: 1$ could be calculated from our data. This confirms the equianalgesic conversion rate of $70: 1$ found by Donner et al. (Donner et al. 1996), ${ }^{5}$ providing a good safety margin to the conversion ratio of 100:1 used in Germany.

\section{Nationwide Survey on Transdermal Fentanyl}

Whereas constipation was less with the transdermal application route than with oral application and other side effects were comparible, there still was considerable concern about the safety of the transdermal system. Reviewers for the German health authorities stated their concern that an incidence of two percent of respiratory depression in cancer pain and up to 6 percent in acute pain (Food and Drug Administration 1990) ${ }^{7}$ was too high to recommend the use of transdermal fentanyl as an alternative for oral morphine, and this had led to the restrictions of the release of transdermal fentanyl in 1995. To find additional data on the safety of the transdermal system a nationwide survey was coordinated in our pain clinic from 1996 to 1998 (Radbruch et al. 2001). ${ }^{8}$ More than 290 physicians participated in this survey, documenting data from 1005 
patients. Most physicians worked in hospital departments and out-patient clinics, though general practitioners participated as well. Pain specialist training was not predominant among these physicians, as only $27 \%$ were members of the German chapter of the International Association for the Study of Pain.

The survey included 996 patients with cancer pain and 11 patients with non-cancer pain. Transdermal therapy was initiated at the time of admission in the survey for 824 patients, while 181 patients had been treated with transdermal fentanyl before admission in the survey. More than half of the patients had been treated with step-3 opioids of the WHO analgesic ladder. However, $23 \%$ had only received pretreatment with step- 2 opioids such as tramadol, $8 \%$ only nonopioid pretreatment and $14 \%$ hat received no analgesics or only as required before initiation of transdermal fentanyl.

The most important reason for switching to transdermal opioid therapy was insufficient pain relief with the previous medication followed by a variety of gastrointestinal symptoms impeding oral analgesic therapy. More than 300 patients were switched from pretreatment with modified release morphine (median dose 90 $\mathrm{mg} / \mathrm{d}$, range $10-950 \mathrm{mg} / \mathrm{d}$ ), and the median conversion ratio to transdermal fentanyl used for these patients was $75: 1$. Normal release morphine and buprenorphine were given less frequently, whereas oxycodone, levomethadone, piritramid or pentazocine were used only rarely. Tramadol and to a lesser extent tilidine were used most frequently as step- 2 opioids, while only a few patients were treated with codeine, dihydrocodeine and dextropropoxyphene. More than $20 \%$ of the patients had received no continuous opioid medication prior to initiation of transdermal therapy.

Transdermal fentanyl therapy was started with a median dosage of $1.2 \mathrm{mg} /$ day $(50 \mu \mathrm{g} / \mathrm{h})$ fentanyl initially, with some patients starting on higher doses up to $9.6 \mathrm{mg} /$ day $(400 \mu \mathrm{g} / \mathrm{h})$. In seven cases transdermal therapy was started with doses of 1.8 or $2.4 \mathrm{mg} /$ day $(75$ or $100 \mu \mathrm{g} / \mathrm{h}$ ), though patients had received no continuous opioid medication before. Most patients needed a dose increase during the study, and the median dosage increased to $2.4 \mathrm{mg} / \mathrm{day}(100 \mu \mathrm{g} / \mathrm{h})$ after 4 months (Fig. 1). The maximum dose given was $21.6 \mathrm{mg}$ fentanyl per day or 9 patches with $100 \mu \mathrm{g} / \mathrm{h}$ each. Only three patients reported that they had difficulties finding enough skin areas for the application due to the high number of patches. The mean duration of transdermal treatment was $71 \pm 83$ days (median 35 days, range 1-478 days, Fig. 1). Transdermal therapy was documented for more than one year for 16 patients.

Initially patches were changed every third day in $95 \%$ of the patients, only $5 \%$ changed patches with other intervals. During the course of transdermal treat-

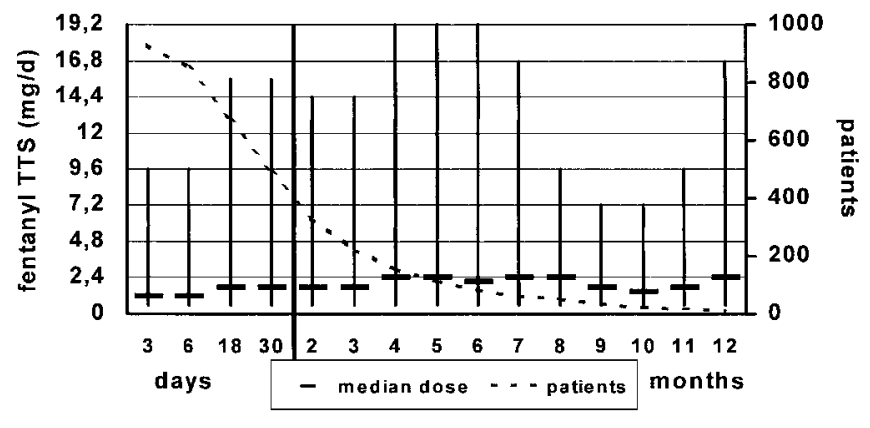

Fig. 1 Fentanyl dosage (median and range) for 1005 patients.

ment application intervals were shortened in some patients. At the end of the study period $14 \%$ of the patients changed patches every second day, and 5\% used other intervals, such as 60-hourly, every fourth day, alternating between every second and third day, or every day. Most patients (up to $70 \%$ on each followup visit) showered regularly with the patches and only three patients reported that patches became loose under the shower or in the bathtub. Problems with transdermal application were reported by $12 \%$ of the patients, with patch detachment $(10 \%)$ and dermatologic symptoms on the site of application (4\%) being most frequent. Seven patients removed a patch themselves, either accidentally while sleeping or during episodes of confusion with advanced disease.

Pain relief with transdermal therapy was swift and efficient (Fig. 2). Whereas half of the patients reported severe or very severe pain on day 0 , the percentage decreased to $14 \%$ on day 3 . The percentage of patients with more than moderate pain remained small during the next months, however, severe pain led to a change of the analgesic regimen in some cases. Episodic pain was documented for $60 \%$ of the patients on day 0 . Although this was reduced considerably during treatment with transdermal fentanyl, episodic pain was reported frequently, often in combination with continuous pain, throughout the study period (Fig. 3).

Adverse events, where the treating physician could not exclude a causal relationship to transdermal therapy, were documented for $26 \%$ of the patients. This included mainly gastrointestinal symptoms such as nausea ( $6 \%$ of the patients), vomiting (3\%) and constipation $(4 \%)$ as well as drowsiness $(3 \%)$, while serious neurotoxic side effects such as hallucinations $(0.2 \%)$, withdrawal $(0.1 \%)$ or convulsions $(0.1 \%)$ were reported only rarely. However, documentation discipline in this open survey may have led to underreport of adverse events. Side effects from the skin were likewise rare with a few cases of erythema $(1 \%)$ and pruritus $(1 \%)$.

Transdermal therapy was continued until the time of death in 424 patients $(42 \%)$. For another 35 patients (4\%) transdermal fentanyl had been discontinued at 


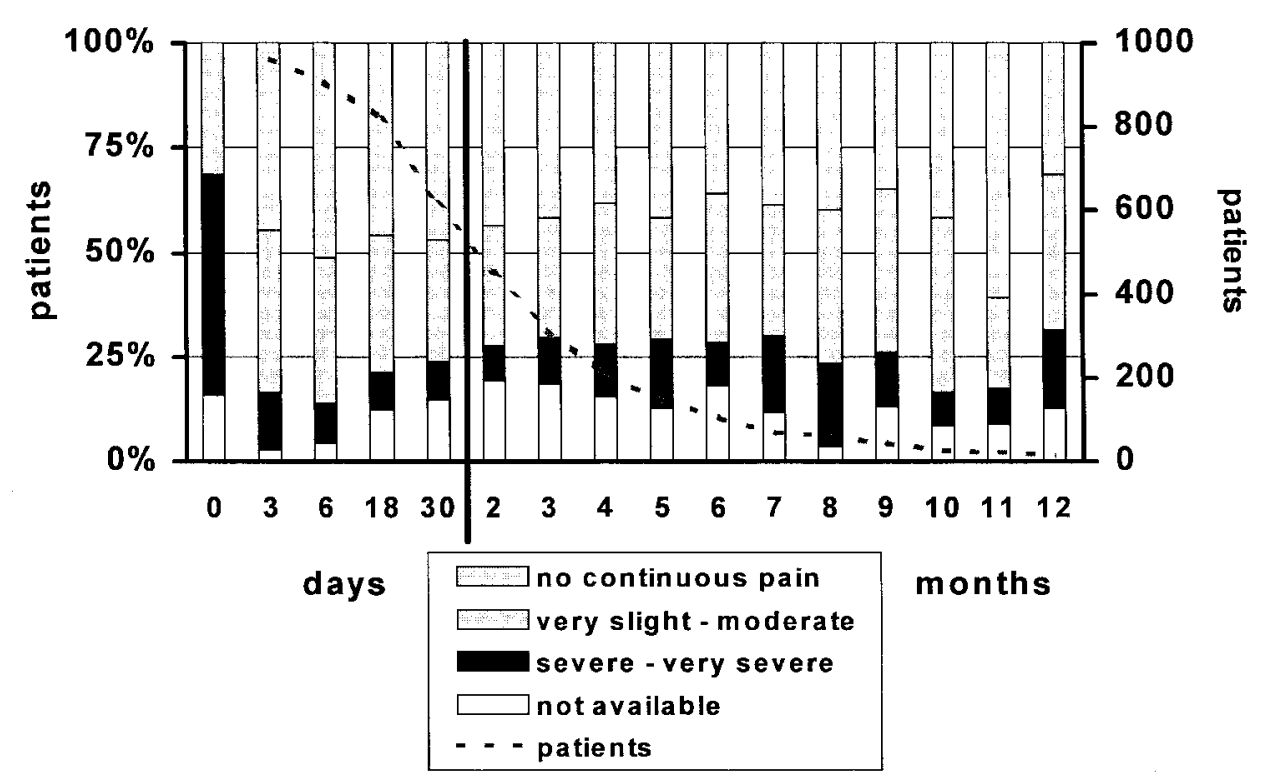

Fig. 2 Intensity of continuous pain before and during transdermal therapy with fentanyl for 1005 patients. Rating by the treating physician on a 5-step descriptive scale (very slight, slight, moderate, severe, very severe).

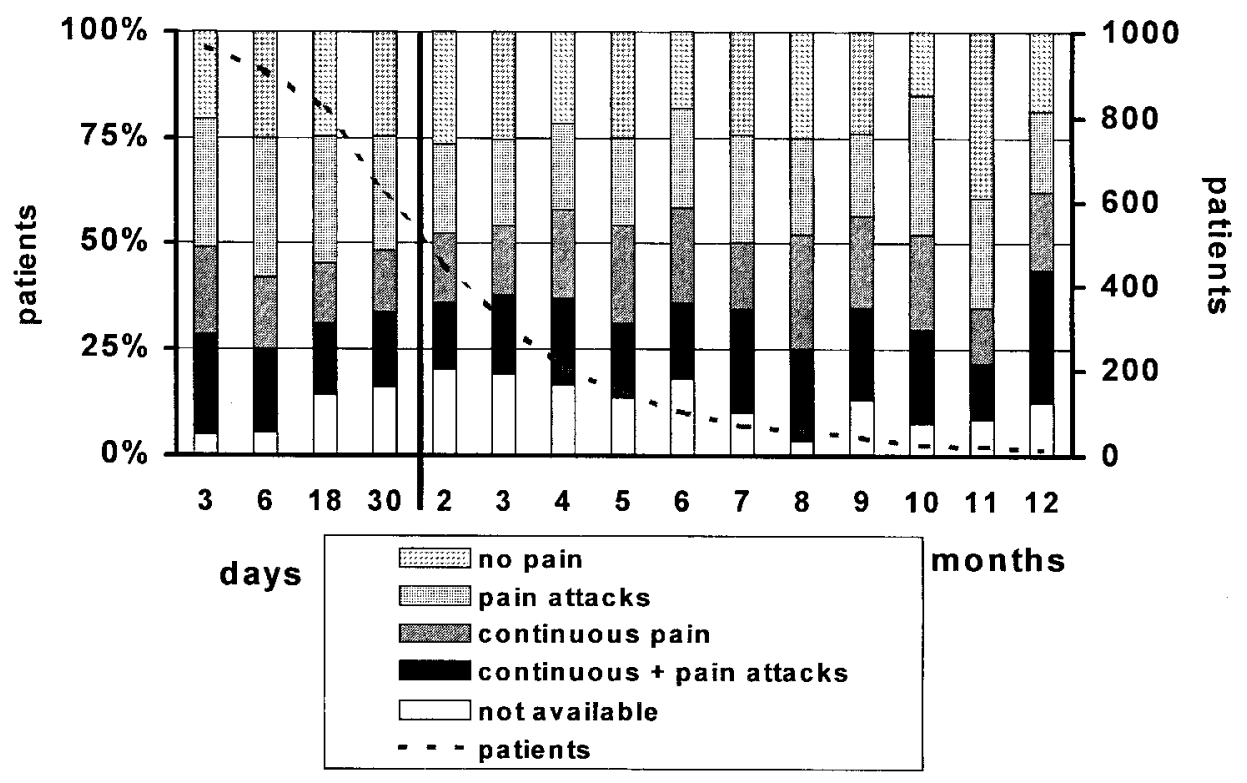

Fig. 3 Prevalence of continuous and episodic pain in 1005 patients treated with transdermal fentanyl. Rating by the treating physician on a checklist (present/not present). For a few patients continuous pain was rated as not present, although intensity of continuous pain (Fig. 2) had been rated as slight or very slight.

least three days before death. Transdermal therapy was discontinued in 355 patients $(35 \%)$ for other reasons than death, most often for inadequate pain relief $(10 \%)$, pain relief with other analgesic regimens $(10 \%)$, adverse events $(5 \%)$, or rejection of transdermal therapy by the patient $(6 \%)$. Respiratory depression, one of the major fears associated with opioid treatment, was reported by the treating physician for 8 patients in the survey. Reviewing these case reports, this may have been caused by progressive disease or concomittant complications such as pleuritis or pnemonia and in most cases probably represented dyspnoea rather than respiratory depression. Dyspnoea is a common symptom in cancer patients and was reported by the patients of the survey in unchanged intensity throughout the observeration period. Dyspnoea is no typical sign of 
opioid-induced respiratory depression, as the opioidrelated decrease of the respiratory drive in the central nervous system usually does not cause the patient to feel breathless. In two cases an overdose was suspected, and both patients needed artifical ventilation for several hours. Both patients had been on stable doses for longer time spans, and the overdose was probably due to progressive renal failure and liver metastases in one patient and pain relief from chemotherapy in the other. Fentanyl is metabolized mainly in the liver and only to a lesser extent in the kidneys and therefore renal failure should not lead to an overdose. However, with decompensation of the renal function and concurrent changes in the fluid balance a redistribution of fentanyl may have led to the overdose. Liver function was not assessed at that time and so the share of the liver metastases in the development of the respiratory depression remained unclear.

Apnoea was reported in a 63 year old patient with chronic lymphatic leucaemia and a history of chronic obstructive lung disease after 6 weeks of transdermal therapy with $1.8 \mathrm{mg}$ fentanyl per day. The patch was removed, and artifical respiration was required for several hours. The patient recovered without sequelae. He had been treated with chemotherapy over the previous 9 weeks, and concurrent pain relief may have led to the observed opioid toxicity.

Side effects from the skin led to discontinuation in three patients. One patient suffered from an allergic reaction to the adhesive and had to stop the application after 10 days. An erythema with exudations and pruritus on the application site was reported in a patient after 56 days of transdermal therapy. At this time the patient was somnolent, and an increase of the fentanyl absorption rate through the damaged skin area may have added to that effect. The third patient discontinued transdermal therapy after three days because of a mild erythema at the site of application.

In the patients of the survey 72 major interventions involving osteosynthesis, laparatomy or thoracotomy were performed during treatment with transdermal fentanyl, and minor interventions such as port implantation, cystoskopy or insertion of a central venous line in 74 cases. Only 18 patients discontinued transdermal therapy in connection with the procedure, in 7 cases because of pain relief from the surgical therapy, in the remaining 11 because of safety concerns. However, transdermal therapy was continued perioperatively without a change of dosage in 111 cases (information not available for 19 patients). This supports our recommendation to leave the transdermal application unchanged perioperatively, if surgical procedures are performed in clinical practice.

In conclusion, in the patients of the survey transdermal therapy with fentanyl was safe and effective, even when analgesic pretreatment and initiation of transdermal therapy did not follow the WHO recommendations and when patients were treated by general practitioners. Most patients continued transdermal therapy until death. Complications were rare, even when patients underwent surgical procedures, or received chemo- or radiotherapy.

\section{Driving Ability and Transdermal Fentanyl}

The use of opioids for the treatment of acute pain is often associated with varying degrees of cognitive impairment. This observation has raised concern that long-term opioid treatment might have a negative impact on cognition and psychomotor function. Clinical experience shows that neuropsychological side effects due to opioid therapy usually decrease in the first weeks under stable medication. However, the effect of longterm treatment with transdermal fentanyl on complex activities such as driving is not yet clear.

In a prospective trial, patients with continuous noncancer pain, who had received stable doses of transdermal fentanyl for at least 2 weeks, completed a series of computerized tests to measure attention, reaction, visual orientation, motor co-ordination and vigilance (Sabatowski R, et al. 2003). ${ }^{9}$ Data were available from 90 healthy volunteers matched to 30 patients, of whom 9 were excluded from the per-protocol analysis because they took additional drugs in violation of the protocol. None of the performance measures for the 21 remaining fentanyl patients was significantly inferior to the controls.

We conclude that stable doses of transdermal fentanyl for the treatment of chronic non-cancer pain are not associated with significant impairments in psychomotor and cognitive performance. The threshold for fitness to drive as defined by German law did not differ significantly between the groups.

\section{Economic Considerations}

In comparing fentanyl-TTS with cr-morphine it is necessary to take into account not only a different safety profile compared with cr-morphine, but also the impact of the different mode of administration on costs and patient functioning. A model was therefore constructed to investigate the cost-utility of transdermal fentanyl compared with controlled release oral morphine in the treatment of moderate-to-severe chronic nonmalignant pain (Lehmann et al. 2002). ${ }^{10}$

The model comprises three phases through which the patients move sequentially: an initial dose titration, subsequent stabilisation and long-term use. The probability and duration of an event, disutility values and unit costs are assigned as appropriate for events occurring 
within each phase. Outcomes associated with each phase, such as the level of pain control, toxicity and quality-adjusted life days, are carried forward to subsequent phases. Use of fentanyl-TTS was predicted to incur higher costs than cr-morphine over 1 year of treatment (Euro 3553 vs Euro 3163, respectively), but was associated with a higher number of quality-adjusted life-days (234 vs 216, respectively), thus achieving an incremental cost-utility ratio of Euro 8160 per qualityadjusted life-year gained. Though there certainly are limitations of using such a model wherein possible inaccuracies are introduced through obtaining estimates from a variety of sources, including assumptions based on expert opinion, the results of the decision-analytic model support the use of fentanyl-TTS as a favourable cost-effective option for the treatment of moderate-tosevere nonmalignant pain.

\section{Opioid Prescriptions in Germany}

The options for opioid therapy have expanded considerably with the introduction of new slow release application forms in Germany. Transdermal application forms of fentanyl (1995) and buprenorphine (2001) as well as oral slow release oxycodone (1998) and hydromorphone (1999) have been released in the last years (Radbruch et al. 2002). ${ }^{11}$

Prescription of WHO-step 3 opioids requires special triplicate prescription forms in Germany. The regulations for these prescription (Betäubungsmittelverschreibungsverordnung BtmVV) have been revised repeatedly. The 10th revision in February 1998 has lifted several restrictions, facilitating the prescription of opioid analgesics. However, physicians still have to order their own prescription forms at the Bundesopiumstelle, the federal regulatory bureau. More than $80 \%$ of the general practitioners reported to have these forms available (Sabatowski et al. 2001). ${ }^{12}$ In another survey $85 \%$ of the general practitioners who had treated cancer patients recently had the prescription forms available, though the percentage was much less for other groups and specialisations of physicians, and only $37 \%$ of the complete sample had opioid prescription forms (Ensink et al. in print). ${ }^{13}$

In the report of drug presciptions for Germany 1.6 million defined daily doses (DDD) of slow release morphine had been prescribed in 1990, and this had increased to 15.4 million DDD in 2000 (Böger and Schmidt 2001; Schmidt 1991). ${ }^{14-15}$ In the same period the amount of other opioids prescribed had increased even more, including buprenorphine (1.3 mio DDD), levomethadone (3.9 mio DDD), oxycodone (7.7 mio DDD) and first of all fentanyl (34.0 mio DDD). Transdermal fentanyl has overtaken morphine in Germany in the number of units and the DDDs sold. These data do not differentiate between prescriptions for nonmalignant and malignant pain, and most of the increases will be related to non-malignant pain. However, in a recent survey morphine and fentanyl had been used as frequently for $25.5 \%$ of the patients each for pretreatment before admission in 1304 patients in German palliative care units. The use of fentanyl was increased even further during inpatient treatment $(28.2 \%$ of the patients, although the use of morphine increased more ( $42.3 \%$ of the patients) (Nauck et al. in print). ${ }^{16}$

\section{Conclusions}

Experience with the transdermal therapeutic system with fentanyl has been gathered in clinical trials, a large nationwide survey and clinical practice since the release in 1995. The conversion table based on a conversion ratio of 100:1 was safe and efficient in trials and clinical practice. Side effects typical for opioids were documented with transdermal application with fentanyl, although constipation was less than with oral morphine. Patients reported ease of administration and high satisfaction with the transdermal therapeutic system. Most patients reported adequate pain relief with one patch system over long periods of time, though some patients have been treated with high dosages up to $1200 \mu \mathrm{g}$ per hour. In some patients, longterm treatment for more than one year with stable dosages has been reported.

Transdermal fentanyl has become a well-known and frequently used opioid in the treatment of chronic cancer and non-cancer pain in Germany.

Acknowledgements: The lecture was supported by a travel grant from Janssen Pharmaceutical K.K., Japan. The chair for palliative medicine of the University Hospital of Aachen (Lukas Radbruch) is funded by an unrestricted grant of the Grünenthal Foundation for Palliative Care.

\section{References}

1. World Health Organisation, Cancer Pain Relief, Geneva, World Health Organisation, 1986

2. Zech DF, Grond SU, Lynch J, Dauer HG, Stollenwerk B, Lehmann KA: Transdermal fentanyl and initial dose-finding with patient-controlled analgesia in cancer pain. A pilot study with 20 terminally ill cancer patients. Pain 1992; 50: 293-301

3. Grond S, Zech D, Lehmann KA, Radbruch L, Breitenbach H, Hertel D: Transdermal fentanyl in the long-term treatment of cancer pain: a prospective study of 50 patients with advanced cancer of the gastrointestinal tract or the head and neck region. Pain 1997; 69: 191-198

4. Ahmedzai S, Brooks D: Transdermal fentanyl versus sustainedrelease oral morphine in cancer pain: preference, efficacy, and quality of life. The TTS-Fentanyl Comparative Trial Group. J Pain Symptom Manage 1997; 13: 254-261

5. Donner B, Zenz M, Tryba M, Strumpf M: Direct conversion from oral morphine to transdermal fentanyl: a multicenter study in patients with cancer pain. Pain 1996; 64: 527-534 
6. Radbruch L, Sabatowski R, Loick G, Kulbe C, Kasper M, Grond S, Lehmann KA: Constipation and the use of laxatives: a comparison between transdermal fentanyl and oral morphine. Palliat Med 2000; 14: 111-119

7. Nightingale SL: From the Food and Drug Administration. JAMA 1990; 264: 1802

8. Radbruch L, Sabatowski R, Petzke F, Brunsch-Radbruch A, Grond S, Lehmann KA: Transdermal fentanyl for the management of cancer pain: a survey of 1005 patients. Palliat Med 2001; 15: $309-321$

9. Sabatowski R, Schwalen S, Rettig K, Herberg KW, Kasper SM, Radbruch L: Driving ability under long-term treatment with transdermal fentanyl. J Pain Symptom Manage 2003; 25: 38-47

10. Lehmann KA, Radbruch L, Gockel HH, Neighbors D, Nuyts G: Costs of opioid therapy for chronic nonmalignant pain in Germany: an economic model comparing transdermal fentanyl (Durogesic) with controlled-release morphine. Eur J Healh Econom 2002; 3: 111-119
11. Radbruch L, Nauck F, Sabatowski R: Germany: Cancer pain and palliative care - current situation. J Pain Symptom Manage 2002; 24: $183-187$

12. Sabatowski R, Arens ER, Waap I, Radbruch L: Cancer pain management in Germany - results and analysis of a questionnaire Schmerz 2001; 15: 241-247 (In Germany)

13. Ensink FBM, Bautz MT, Voß MC, Görlitz A, Hanekop GG: Cancer pain management in Germany - results and analysis of a questionnaire. Schmerz; in print (In Germany)

14. Böger RH, Schmidt G: Analgetika. In: Schwabe, U, Paffrath D, eds, Arneiverordnungsreport 2001, Berlin, Springer, 2001; 89102

15. Schmidt G: Analgetika. In: Schwabe U, Paffrath D, eds, Arneiverordnungsreport 1991, Stuttgart, Gustav Fischer Verlag, 1991; 20-31

16. Nauck F, Ostgathe C, Radbruch L, Bausewein C, Fuchs M, Lindena G, Neuwöhner K, Schulenberg D, Klaschik E: Drugs in Palliative Care - Results from a respresentative survey, Palliative Medicine; in print 